\title{
Quality Improvement in Sweet Red Wines Through an Alternative Grape-Drying System
}

\author{
A. Marquez, M.P. Serratosa and J. Merida* \\ Department of Agricultural Chemistry, Faculty of Sciences, University of Cordoba, Bd. Marie Curie, Campus of Rabanales, \\ E-14014, Cordoba, Spain
}

Submitted for publication: February 2013

Accepted for publication: July 2013

Key words: Sweet red wines, phenolic compounds, anthocyanins, colour, sensorial analysis

\begin{abstract}
A sensorial analysis was undertaken and the colour parameters and phenolic profile were measured for two types of Andalusian sweet red wines. Two wines types were studied, i.e. commercial and alternative wines elaborated with musts from grapes obtained by traditional sun drying and by chamber drying under controlled temperatures respectively. Results show that the alternative wines obtained in this study were analytically closer to typical red wines in their lower concentration of browning compounds, a high contribution of red and blue colour, and hence having a more suitable hue than the traditional sweet wines. Furthermore, the alternative wines were the richest in monomeric anthocyanins, flavan-3-ols and flavonols, which give these wines their antioxidant properties. Their sensorial characteristics were accepted by consumers, which means that the alternative grape-drying system improves the quality of sweet red wines relative to those obtained by the traditional method.
\end{abstract}

\section{INTRODUCTION}

Wine quality is influenced by several factors, such us the health of the grapes and their chemical composition. In relation to the last, in addition to the sugar concentration and acidity it is necessary to know the concentrations of phenolic compounds present in the berries, which will depend on various factors such as grape variety (Perez-Magariño et al., 2009), maturation degree, culture practices (Downey et al., 2006; Petropoulos et al., 2011), and environmental factors such as soil (Prado et al., 2007) and climate (Jackson \& Lombard, 1993).

The importance of the phenolic composition in oenological products and their relation to quality is because these compounds are responsible for the organoleptic characteristics such as bitterness and astringency (Fischer \& Noble, 1994; McRae \& Kennedy, 2011; Chira et al., 2012), as well as for the colour of musts and wines (Glories, 1984; Garcia-Puente Rivas et al., 2006; Monagas et al., 2006). Moreover, the interest in this chemical family is based on its antioxidant properties (Alen-Ruiz et al., 2009; Sun et al., 2011), responsible for the "French paradox" and the prevention of cardiovascular diseases and cancer, among others (Vidavalur et al., 2006).

To obtain sweet wines, the grapes can be subjected to different processes prior to winemaking, like drying processes to dehydrate the berries, the off-vine system, which is the most common. Dehydration is a complex process that increases the sugar content, but induces several changes in the physicochemical characteristics of grape berries. Firstly, the evaporation of water involves significant changes in the cellular structure of the skins, which lose elasticity, increasing the risk of breakage. The raisins therefore are characterised by a loss of firmness and softening of their texture (Rolle et al., 2012), which increase their susceptibility to mechanical damage and infection by pathogens (Genovese et al., 2007).

On the other hand, grape dehydration leads to important modifications in colour, volatile compounds and phenolic composition (Bellicontro et al., 2004; Serratosa et al., 2008a). In particular, the phenolic compounds present in the skins are diffused to the pulp due to their structural alterations (Marquez et al., 2012b). These compounds participate in several types of reactions, resulting in the disappearance of some of them and the formation of others. Furthermore, because of the cellular damage, the phenolic compounds contained in the vacuoles of the plant cells come into contact with the polyphenol oxidase enzymes in the cytoplasm ( $\mathrm{Li}$ et al., 2008). Consequently, the enzymatic reactions begin in the presence of oxygen and the brown compounds, called melanins, are synthesised. All these reactions induce a colour change in musts and in elaborated wines. Due to changes in the phenolic composition in the grapes during the dehydration process, the final wines obtained also present different concentrations of phenolic compounds.

Natural sun drying is still the most commonly used

*Corresponding author: E-mail: jmerida@uco.es [Tel.: +34 957 218612; Fax: +34 957 212146]

Aknowledgements: The authors gratefully acknowledge financial support from the Spanish Government, via the Minister of Education (FPU scholarship for Ana Marquez), for the realisation of this work 
method for dehydrating grapes in the Mediterranean region as a result of the large number of sun hours. In this raisining process, grapes are exposed to sun on mats for five to ten days to increase their sugar content (Serratosa et al., 2008b; Ruiz et al., 2010; Serratosa et al., 2012). This traditional method is widely used in the Montilla-Moriles region (southern Spain) to obtain sweet white wines. However, in the case of sweet red wines, this drying system decreases the quality, mainly due to the loss of the typical red colour of these wines.

Under this premise, the aim of this work was to elaborate sweet red wines by an alternative off-vine drying system to improve their quality in relation to other commercial sweet red wines obtained by traditional sun drying and produced in the Montilla-Moriles region. The wines were compared by analysing their sensory profile, colour parameters and phenolic composition.

\section{MATERIALS AND METHODS \\ Reagents}

Methanol, formic acid, hydrochloric acid and acetonitrile were purchased from Merck (Madrid, Spain). Phenolic commercial standards were purchased in 95 to $99 \%$ purity from Sigma-Aldrich Chemical Co. (Madrid, Spain) and Extrasynthèse (Genay, France).

\section{Commercial traditional wines}

Three samples $(a, b, c)$ of each commercial sweet red wine (w1 and w2) obtained from two different winemakers in the Montilla-Moriles region (southern Spain) were subjected to analyses. These wines were chosen for being the only commercial sweet red wines made from a sun-drying process in the Montilla-Moriles region. The wines were obtained from $15 \%(\mathrm{v} / \mathrm{v})$ fortified musts by the addition of wine alcohol. W1 and w2 were elaborated with Syrah/Tempranillo and Merlot grapes respectively, which were subjected to a traditional sun-drying process in order to raisin the berries.

\begin{abstract}
Alternative vinification
The grapes used were Vitis vinifera L. cv. Merlot, Syrah and Tempranillo from the 2009 harvest in the Montilla-Moriles region. A total of about $30 \mathrm{~kg}$ of grapes was uniformly distributed in a single layer in several trays (11 to $19 \mathrm{~kg}$ / $\mathrm{m}^{2}$, depending on the variety), and dried off-vine in a Frisol Climatronic chamber at a constant temperature of $40^{\circ} \mathrm{C}$ and an initial relative humidity of $20 \%$. The drying process was finished when the reducing sugar content reached approximately $31.4^{\circ} \mathrm{Brix}$. Once dried, the raisins were crushed in a vertical press similar to industrial models, using a maximum pressure of 300 bar. The resulting musts were fortified to $15 \%(\mathrm{v} / \mathrm{v})$ with wine alcohol (no. CE 200-5786, Alcoholes del Sur, SA) and macerated with the skins for $47 \mathrm{~h}$ at $25^{\circ} \mathrm{C}$. After maceration, the grapes were pressed and the skin residues were removed from the wine. The resulting wines (w3, w4 and w5) were centrifuged at $3000 \mathrm{rpm}$, filtered and analysed as described below.
\end{abstract}

\section{Analytical determinations}

${ }^{\circ}$ Brix was measured using an Atago Master refractometer (Master Baume 2594, Atago, Japan).

\section{Colour parameters}

Spectrophotometric measurements were made with a PerkinElmer (Waltham, MA) Lambda 25 spectrophotometer, using quartz cells with a $1 \mathrm{~mm}$ light path. Samples were previously passed through Millipore (Billerica, MA) HA filters of $0.45 \mu \mathrm{m}$ pore size, and all measurements were corrected for a path length of $1 \mathrm{~cm}$. Absorbances at 420, 520 and $620 \mathrm{~nm}$ were measured and the percentages of yellow, red and blue colour were calculated. Hue was calculated as the relationship between absorbance at $420 \mathrm{~nm}$ and absorbance at $520 \mathrm{~nm}$. Polymeric pigment colour (PPC) was obtained as the absorbance at $520 \mathrm{~nm}$ of $5 \mathrm{~mL}$ of sample previously supplied with $15 \mathrm{mg}$ of $\mathrm{NaHSO}_{3}$ and allowed to stand at $25^{\circ} \mathrm{C}$ for 45 minutes.

\section{Analysis of phenolic compounds}

For the extraction and fractionation of anthocyanins, the method proposed by Marquez et al. (2012b) was used. A volume of $2 \mathrm{~mL}$ of wine was passed through a Sep-Pak C18 cartridge packed with $900 \mathrm{mg}$ of material (Long Body Sep-Pak Plus, Water Associates, Milford, MA) that was previously activated with $5 \mathrm{~mL}$ of methanol and washed with aqueous $0.01 \%(\mathrm{v} / \mathrm{v}) \mathrm{HCl}$. The cartridge was successively washed with $10 \mathrm{~mL}$ of $0.01 \%$ aqueous $\mathrm{HCl}$ and $5 \mathrm{~mL}$ of ethyl acetate, and the anthocyanins were recovered with $5 \mathrm{~mL}$ of methanol acidified to $\mathrm{pH} 2$ with $\mathrm{HCl}$. Anthocyanin samples (fraction 1) were concentrated on a vacuum centrifuge thermostated at $35^{\circ} \mathrm{C}$ and passed through a filter of $0.45 \mu \mathrm{m}$ pore size for injection into a P4000 HPLC instrument from Spectra-Physics (San Jose, CA).

Other phenolic compounds were extracted with a combination of two cartridges to ensure effective separation (Marquez et al., 2012b). Thus, a volume of $15 \mathrm{~mL}$ of wine was adjusted to $\mathrm{pH} 7$ with $0.1 \mathrm{M} \mathrm{NaOH}$ and passed through Sep-Pak C18 cartridges previously activated with $5 \mathrm{~mL}$ of methanol and washed with distilled water, which was adjusted to $\mathrm{pH} 7$ with $\mathrm{NaOH}$. The cartridges were eluted with $10 \mathrm{~mL}$ of water at $\mathrm{pH} 7$. This volume, in addition to the volume obtained as a result of sample run-through prior to elution, was used to determine hydroxybenzoic acids and hydroxycinnamic esters (fraction 2). After preconditioning of the cartridge with $5 \mathrm{~mL}$ of water at $\mathrm{pH} 2$, flavan3-ols (fraction 3) were eluted with $40 \mathrm{~mL}$ of $16 \%$ acetonitrile in water at $\mathrm{pH} 2$. Flavonols (fraction 4) were eluted with $5 \mathrm{~mL}$ of ethyl acetate, evaporated to dryness and redissolved in methanol. All collected fractions were concentrated on a vacuum centrifuge thermostated at $35^{\circ} \mathrm{C}$ and passed through a filter of $0.45 \mu \mathrm{m}$ pore size for injection into the SpectraPhysics P400 HPLC instrument.

All the phenolic compounds were identified by comparing their retention times with those for standards, recording UV spectra on a Spectra-Physics UV6000LP diode array spectrophotometer and calculating the UV absorbance ratios for samples and standards simultaneously co-injected one at a time (Marquez et al., 2012b). Identification was confirmed by HPLC-ESI-MS on an AQA quadrupole mass spectrometer from ThermoQuest Finningan. The instrument was operated in both the negative and positive ion modes. The ion spray voltage was $-4 \mathrm{kV}$ and the orifice voltage was $-60 \mathrm{~V}$. Mass data were acquired in the scan mode (by 
scanning the $\mathrm{m} / \mathrm{z}$ range 150 to 1066 at 1.2 intervals) and the multiple ion mode (by using mass ranges around specific $\mathrm{m} / \mathrm{z}$ values).

Each compound was quantified by comparison with a calibration curve obtained from the corresponding standard, except for caftaric, coutaric and fertaric acid, which were quantified as caffeic, $p$-coumaric and ferulic acid respectively; procyanidins were quantified as $(+)$-catechin; quercetin-3-glucuronide, laricitrin-3-glucoside, syringetin3-glucoside, isorhamnetin-3-glucoside and isorhamnetin were quantified as quercetin-3-glucoside; kaempferol-3glucoside and kaempferol-3-glucuronide were quantified as kaempferol; and anthocyanins were quantified as malvidin3-glucoside.

\section{Statistical procedures}

The results for all samples were subjected to principal component analysis (PCA), using the Statgraphics Computer Package v. 5.0 from Statistical Graphics Corp.

\section{Sensory analysis}

The wines were assessed for aroma, flavour and colour acceptability by 15 experts in a panel in accordance with ISO 8586-1:1993. The selected tasters were: two professional oenologists (male, 40 to 50 years old) selected by the Quality Regulation Board of the Montilla-Moriles Designation of Origin (Southern Spain), seven oenologists (two male, 45 to 55 years old, and five female, 30 to 40 years old) and six consumers who know the characteristics of typical wines produced in this region.

The tasting room was kept at $20^{\circ} \mathrm{C}$ and had individual booths. Standardised glasses filled with $20 \mathrm{~mL}$ of wines were used in accordance with ISO 3591:1992. The glasses were well marked with a code and covered to avoid any loss of organoleptic properties. All the samples were evaluated in a single session, one at a time and with a wait of three minutes between samples. The tasters were instructed in advance about their task and the rules to be followed, and were given a scoring sheet. The evaluation of the quality wines was scored using the scale methods according to ISO
4121:2003, with values of desirable (5 \& 6), acceptable ( $3 \&$ 4) and undesirable ( $1 \& 2)$.

\section{RESULTS AND DISCUSSION}

The commercial sweet red wines obtained from traditional sun-dried grapes (w1 and w2) are currently on the market and widely accepted by consumers. However, the alternative wines from the chamber-dried grapes (w3 to w5) are not commercially available. The first step was to evaluate the sensory differences between them with a view to assessing the customer acceptance potential of the alternative wines. A bottle of each type of wine was used to analyse the aroma, flavour and colour, which were scored on the following acceptance scale: undesirable (scores 1 \& 2), acceptable (3 $\& 4$ ) and desirable ( $5 \& 6)$. As can be seen in Fig. 1, w1 was judged to have the best aroma (average score 3.9). The other wines received slightly lower scores, but exhibited no significant differences between grape-drying methods. The flavour scores were more different between the wines, with higher values for the alternative wines (3.6 for w5 and 4.4 for w3). Finally, the single property that best differentiated the two wines types was the colour. Thus, the scores for traditional wines (2.2 to 2.8) were substantially lower than those for the alternative ones (3.7 to 4.4). Therefore, these wines could have the potential to compete with the commercial wines and an analytical study consequently was undertaken.

Table 1 shows the contents of the alternative and traditional sweet red wines in low molecular weight anthocyanins, which were identified and quantified by HPLC-DAD-MS. As can be seen, there were qualitative and quantitative differences between the two wine types. Thus, the traditional wines (w1 and w2) did not contain any monomeric anthocyanins. By contrast, the three alternative wines (w3 to w5) contained monomeric glycosylated and acetylglycosylated derivatives of the following anthocyanidins: delphinidin, cyanidin, petunidin, peonidin and malvidin; the coumaroyl glycosides of cyanidin, petunidin, peonidin and malvidin; and malvidin-3-caffeoylglucoside. In addition, the analyses revealed the presence of other anthocyanin derivatives with

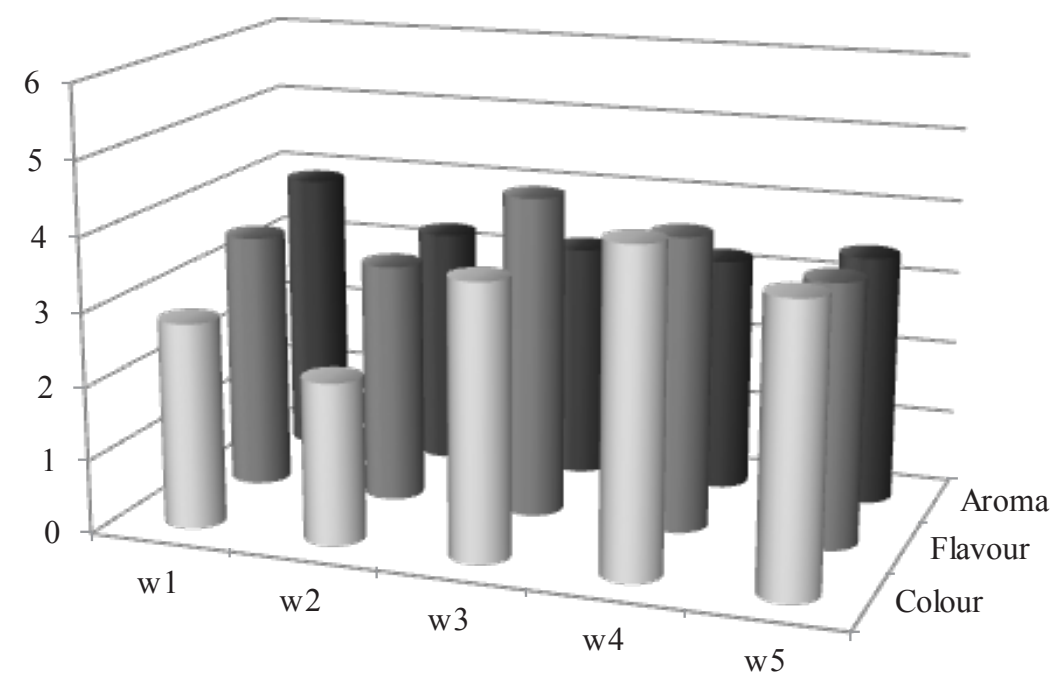

FIGURE 1

Sensorial analysis of the two types of sweet red wines: commercial wines (w1 and w2) and alternative wines (w3 to w5). 


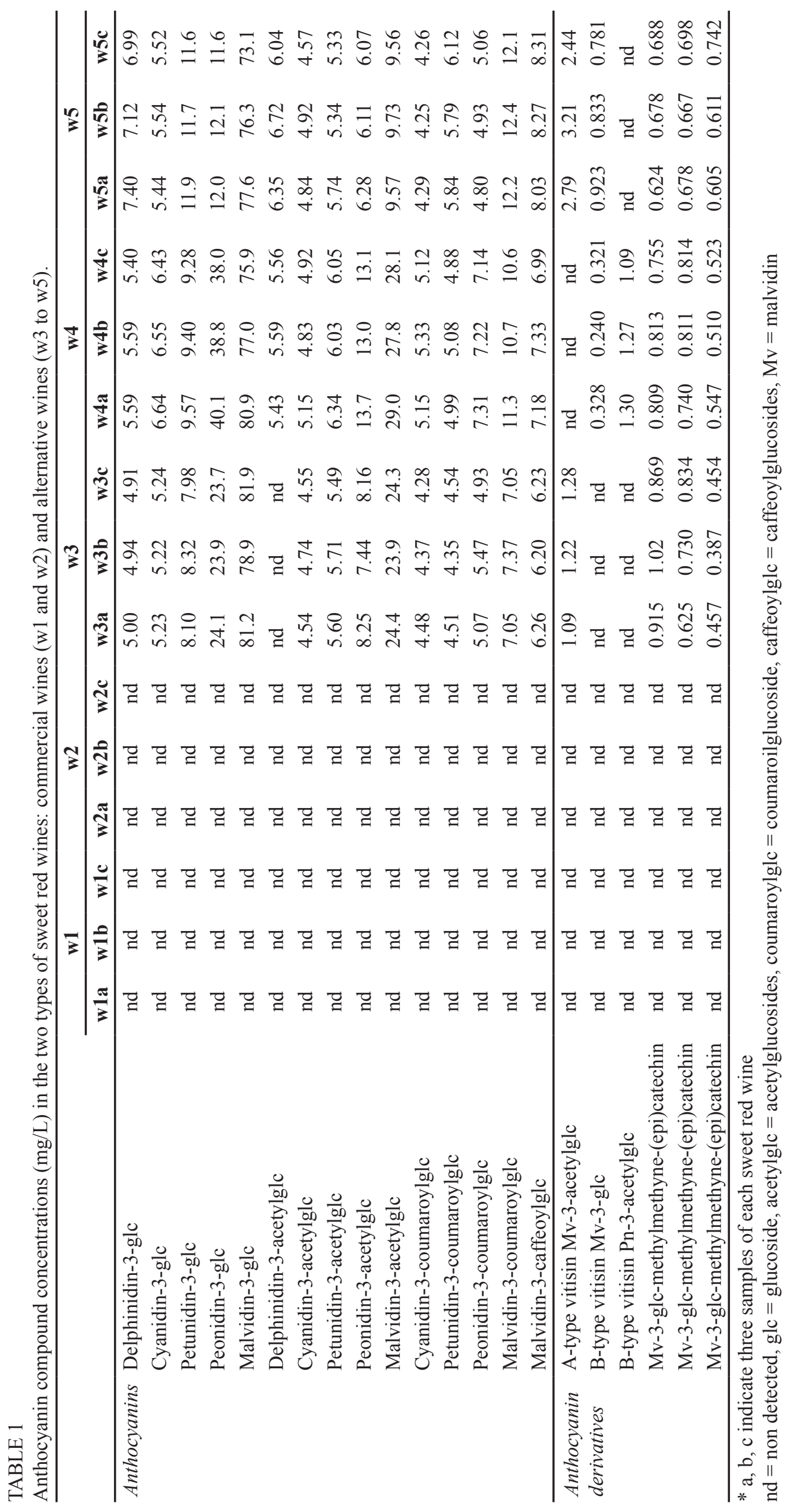


pyranoanthocyanin structures and malvidin-3-glucoside and (epi)catechin adducts bonded via a methylmethyne bridge. The complete absence of anthocyanins in the traditionally made wines does not necessarily mean that no anthocyanins were extracted. In fact, cellular damage of the grapes as a result of dehydration caused the diffusion of coloured compounds from the berry skin to the pulp in all grape-drying processes, and this may occur to a greater or lesser extent depending on the drying system. Therefore it is reasonable to assume that monomeric anthocyanins were extracted during the drying of the grapes and, subsequently, that they could take part in various reactions such as degradation, polymerisation and, possibly, browning.

The most likely polymerisation reactions in the sweet red wines could involve the monomeric anthocyanins and tannins to form coloured compounds of high molecular weights, increasing the temporal stability of their colour (Soto-Vazquez et al., 2010). The formation of these products was studied via polymeric pigment colour (PPC). As can be seen in Fig. 2, the traditional wines, which did not contain monomeric anthocyanins, exhibited greater amounts of polymeric pigments. This could confirm that monomeric anthocyanins are involved in the polymerisation reactions, leading to the formation of coloured compounds. The PPC values for the alternative wines also indicate the formation of pigments, albeit to a lesser extent than in the traditional wines.

The abovementioned monomeric anthocyanins could also take part in browning reactions. To this end, the absorbances of the wines at $420 \mathrm{~nm}$ (A420) were measured. As can be seen in Table 2, the traditional wines exhibited a much higher browning index than the alternative wines, without significant differences between them. All wines had been made according to the same winemaking process, except for the grape-drying step. Consequently, the sun drying must have substantially enhanced the synthesis of brown compounds, which absorb at this wavelength. Thus, the A420 values for the traditional wines were approximately double (w2) and triple (w1) those of the all alternative wines (w3 to w5). These results are consistent with others previously found by Serratosa et al. (2008a) in musts from sun-dried and chamber-dried Pedro Ximenez grapes. The increased progress of browning in the traditional wines can be ascribed to several factors. On the one hand, sun drying takes longer (seven to 10 days) than the time used for the alternative wines (two to three days).

On the other hand, sun-dried grapes are subject to abrupt temperature changes, where the berry surface can reach high temperatures at midday (Serratosa et al., 2008b) because the temperature can reach $40{ }^{\circ} \mathrm{C}$ around midday (Lopez et al., 1988), whereas nocturnal values frequently fall below $18^{\circ} \mathrm{C}$ and are accompanied by dew. A constant temperature was utilised in the chamber-drying process.

One hypothesis to explain the differences in the formation of brown compounds might not be due to the temperature value, but the use of temperature changes or constant temperature. So a high temperature results in the continuous evaporation of water from the berries, which hinders oxygen penetration through their skins. However, when the temperature falls, the water evaporation stops and atmospheric oxygen again penetrates the skin, as the solubility is more favourable at low temperatures. The constant temperature $\left(40^{\circ} \mathrm{C}\right)$ used in the chamber-drying process facilitates the sustained evaporation of water from the pulp, thereby completely hindering the penetration of gases (particularly oxygen). The fact that oxygen is required by many browning reactions may account for its different progression in the two types of processes. On the other hand, the undesirable browning reactions potentially occurring in red wines therefore could be non-enzymatic (Maillard reaction, autoxidation, etc.) and enzymatic types through the action of polyphenol oxidases (PPOs) on phenolic compounds. However, Maillard reactions would not be favoured, since the temperature never reached $50^{\circ} \mathrm{C}$ during the process (Rivero-Perez et al., 2002). As a result of the anthocyanin compounds being involved in the browning reactions, the wines exhibiting the greatest levels of browning were those without monomeric anthocyanins.

Despite the absence of monomeric anthocyanins, the traditional wines exhibited measurable values of A520, which, however, were lower than those of the alternative wines (Table 2). These values in the traditional wines

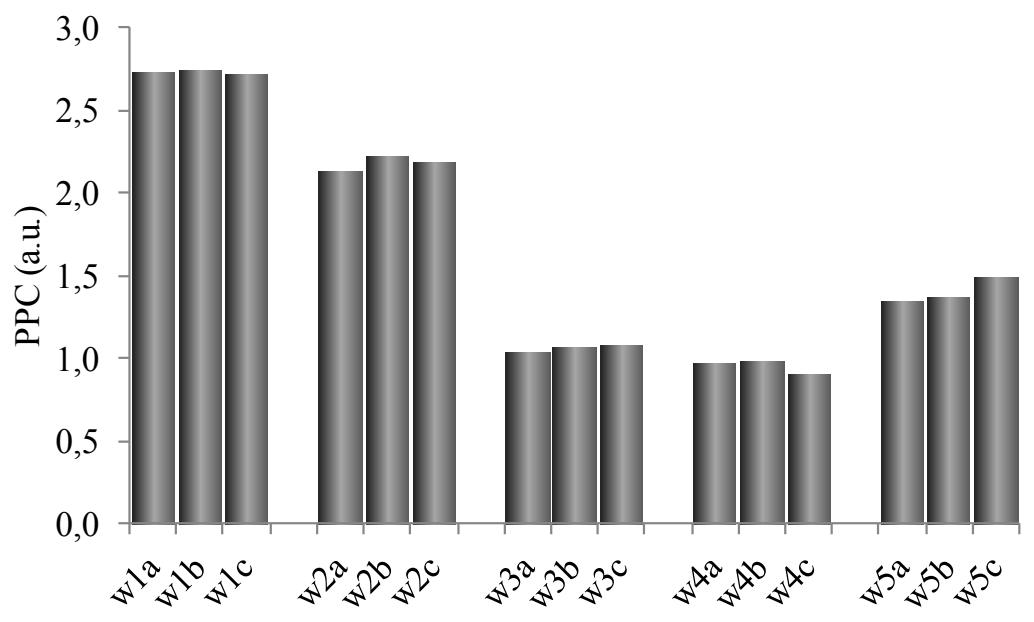

FIGURE 2

Polymeric pigment colour (PPC) in the two types of sweet red wines: commercial wines (w1 and w2) and alternative wines (w3 to w5). 
must have been the result of the contribution of polymeric pigments absorbing at $520 \mathrm{~nm}$ and the high concentration of brown compounds, which absorb maximally at $420 \mathrm{~nm}$ but also, less strongly, at $520 \mathrm{~nm}$.

Wine hues (A420/A520) are shown in Fig. 3. As can be seen, this parameter greatly exceeded unity in the traditional wines $(>2.43)$, suggesting that brown hues clearly exceeded red ones. On the other hand, the alternative wines had hue values less than unity $(<0.732)$, exhibiting a typical red colour not present in the traditional wines. Therefore, the use of an alternative grape-drying method may improve the quality of sweet red wines.

Colour differences between the wines were also observed in their percentages of yellow, red and blue. As can be seen in Table 2, and contrary to the expectations for red wines, $\%$ yellow prevailed in the commercial wines (64.6 to 68.9\%), where it considerably exceeded \% red (23.6 to $26.6 \%)$. On the other hand, $\%$ red prevailed in the three alternative wines $(>51 \%)$. Finally, $\%$ blue was the least in all the wines $(7.63$ to $10.1 \%)$.

In relation to the anthocyanin concentrations in the alternative wines (Table 1), glycosylated derivatives were the major compounds, with slight differences between them. Specifically, peonidin-3-glucoside was found at concentrations from $11.6 \mathrm{mg} / \mathrm{L}$ in w $5 \mathrm{c}$ to $40.1 \mathrm{mg} / \mathrm{L}$ in w $4 a$. With respect to the acetyl glycosylated derivatives, that of delfinidin was not found in any w3 samples. The peonidin derivative was again the compound that differed most markedly between wines, possibly as a result of the different grape varieties used. Finally, the coumaroyl glycosylated derivatives were found at very low concentrations relative to the previous compounds.

In addition to these anthocyanins, the alternative wines contained pyranoanthocyanin compounds and adducts of malvidin-3-glucoside and (epi)catechin bonded by a methylmethyne bridge. Although all were found at very low concentrations (less than $1 \mathrm{mg} / \mathrm{L}$ in most cases), their presence in sweet red wines is interesting, since they protect them from $\mathrm{pH}$ changes and decolourisation by $\mathrm{SO}_{2}$ (Fulcrand et al., 1998; Escribano-Bailon et al., 2001; He et al., 2010). Furthermore, these compounds increase the temporal colour stability (Bakker et al., 1993). The alternative wines were not subjected to fermentation, so pyruvic acid or acetaldehyde, which are indispensable for anthocyanin derivative formation, were not produced by the yeasts. The presence of these two compounds has been ascribed to various enzymatic reactions during grape drying in a controlled-temperature chamber (Marquez et al., 2012a). As a result of the presence of these compounds, colour stabilisation in the sweet red wines from chamber-dried grapes could be assumed to be an additional quality factor for these alternative wines.

The non-flavonoid fraction of the wines included four hydroxybenzoic acids, three hydroxycinnamic acids and the tartaric esters of the latter (Table 3 ). In relation to the hydroxybenzoic acids, gallic, vanillic and syringic acids were present in the five types of wines, whereas protocatechuic acid

TABLE 2

Colour parameters in the two types of sweet red wines: commercial wines (w1 and w2) and alternative wines (w3 to w5).

\begin{tabular}{|c|c|c|c|c|c|c|c|c|c|c|c|c|c|c|c|}
\hline & \multicolumn{3}{|c|}{ w1 } & \multicolumn{3}{|c|}{ w2 } & \multicolumn{3}{|c|}{ w3 } & \multicolumn{3}{|c|}{ w4 } & \multicolumn{3}{|c|}{ w5 } \\
\hline & w1a & w1b & w1c & w2a & $\mathbf{w} 2 \mathrm{~b}$ & w2c & w3a & $\mathbf{w b}$ & w3c & w4a & w4b & $w 4 c$ & w5a & w5b & w5c \\
\hline A420 (a.u.) & 9.06 & 9.03 & 9.10 & 5.81 & 5.84 & 5.83 & 3.02 & 3.06 & 3.02 & 2.78 & 2.79 & 2.79 & 2.87 & 2.85 & 2.87 \\
\hline A520 (a.u.) & 3.09 & 3.08 & 3.14 & 2.39 & 2.40 & 2.40 & 4.13 & 4.18 & 4.11 & 4.55 & 4.54 & 4.56 & 3.92 & 3.90 & 3.91 \\
\hline A620 (а.и.) & 1.00 & 1.01 & 1.04 & 0.790 & 0.786 & 0.797 & 0.797 & 0.811 & 0.795 & 0.702 & 0.702 & 0.708 & 0.755 & 0.747 & 0.752 \\
\hline$\%$ Yellow & 68.9 & 68.8 & 68.5 & 64.6 & 64.7 & 64.6 & 38.0 & 38.0 & 38.1 & 34.6 & 34.8 & 34.6 & 38.1 & 38.0 & 38.1 \\
\hline$\%$ Red & 23.5 & 23.5 & 23.6 & 26.6 & 26.6 & 26.6 & 52.0 & 51.9 & 51.9 & 56.6 & 56.5 & 56.6 & 51.9 & 52.0 & 51.9 \\
\hline$\%$ Blue & 7.63 & 7.72 & 7.83 & 8.79 & 8.71 & 8.83 & 10.0 & 10.1 & 10.0 & 8.74 & 8.75 & 8.79 & 10.0 & 9.97 & 9.97 \\
\hline
\end{tabular}

* a, b, c indicate three samples of each sweet red wine

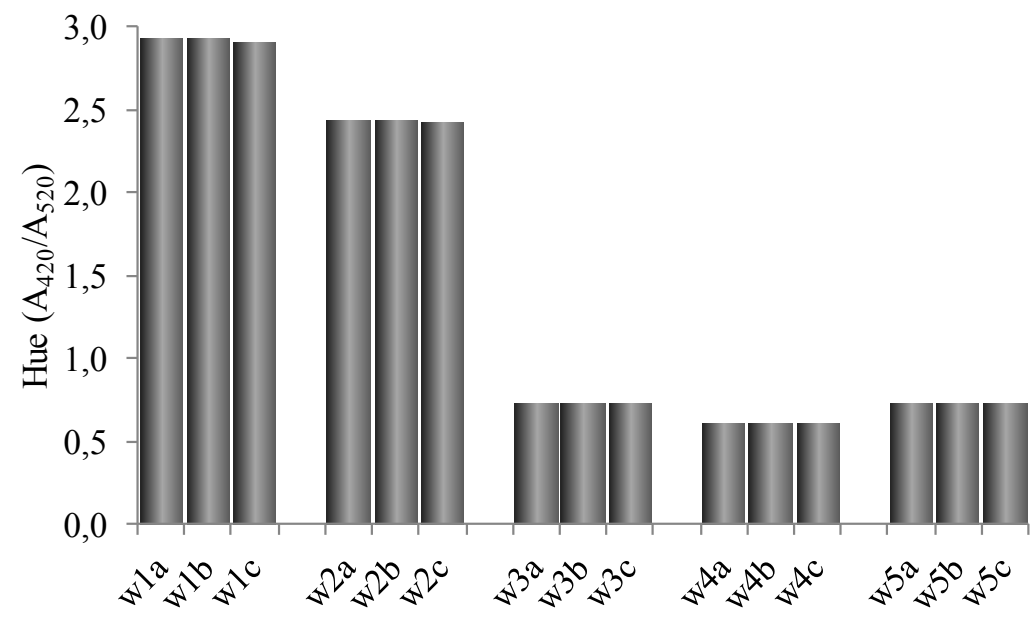

FIGURE 3

Hue (A420/A520) in the two types of sweet red wines: commercial wines (w1 and w2) and alternative wines (w3 to w5). 


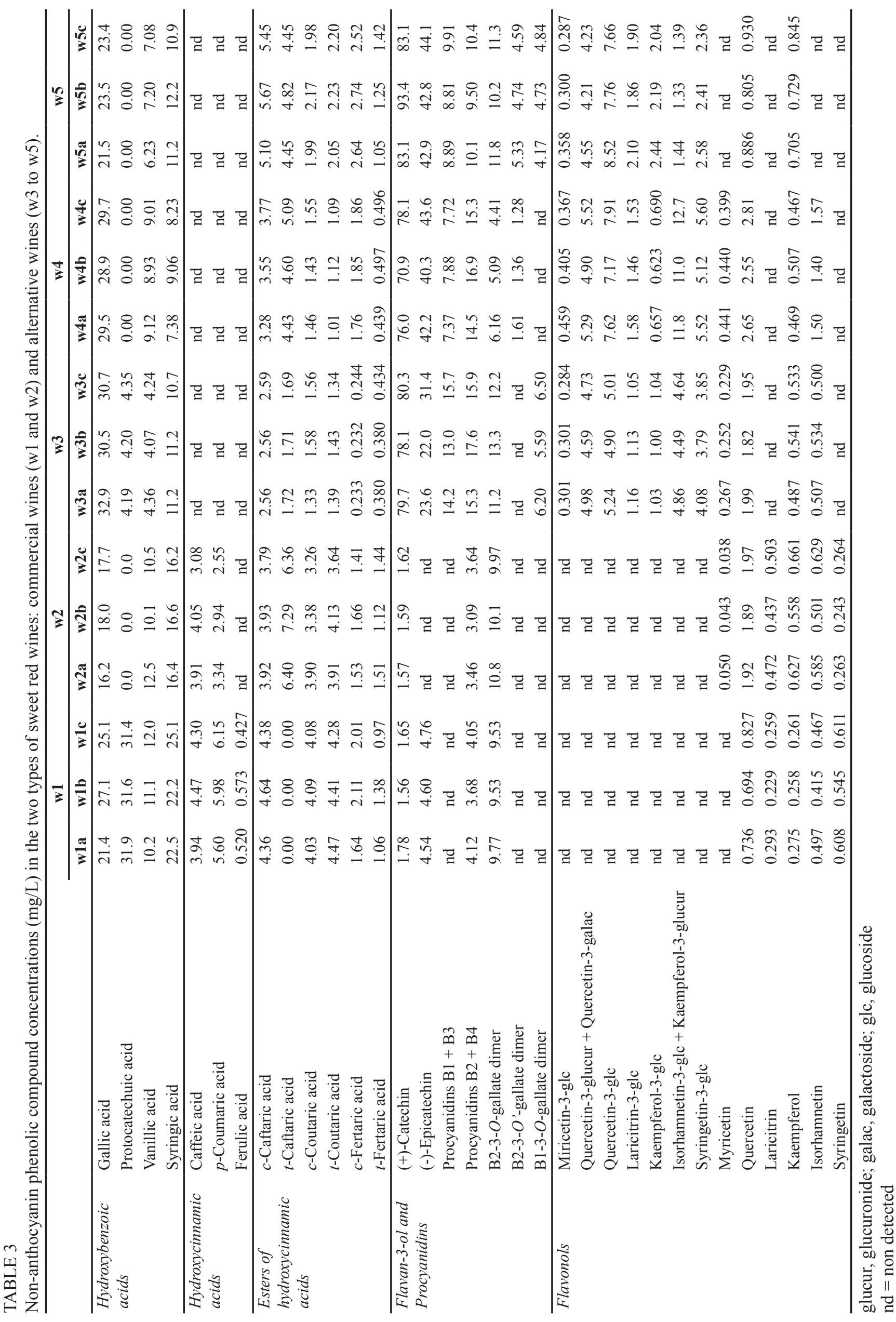


was present only in the three $\mathrm{w} 1$ wines. The concentrations of gallic acid ranged from $16.2 \mathrm{mg} / \mathrm{L}$ (w2a) to $32.9 \mathrm{mg} / \mathrm{L}$ (w3a), without any significant differences in terms of the drying method or grape variety. On the other hand, differences in the concentrations of vanillic and syringic acids were found relative to the vinification methods, since both compounds exhibited somewhat higher concentrations in the w1 and w2 type wines (traditional wines).

The hydroxycinnamic acids caffeic, p-coumaric and ferulic were found in $\mathrm{w} 1$ and w2 wines, although none was detected in the alternative wines (Table 3). The low concentrations of the three acids in $\mathrm{w} 1$ and $\mathrm{w} 2$ (below $7 \mathrm{mg} / \mathrm{L}$ ) suggest that the difference in the alternative wines depended on the initial raw material. The concentrations in berry phenolic compounds and in the resulting wines are known to depend on the grape variety, cultural practices and environmental factors (Downey et al., 2006; Petropoulos et al., 2011).

The tartaric esters of the three hydroxycinnamic acids were detected in all the wines. As can be seen in Table 3, caftaric and coutaric acids were present at higher concentrations than fertaric acids, the cis isomers of which generally prevailed over the trans isomers. Caftaric acids were found at concentrations from 2.56 to $5.67 \mathrm{mg} / \mathrm{L}$, without differences in terms of the grape-drying method. Finally, the coutaric acid concentrations in the traditional wines were above $3.26 \mathrm{mg} / \mathrm{L}$ and exceeded those in the alternative wines.

The low molecular weight flavan-3-ol compounds are especially important in red wines because their polymerisation reactions lead to tannins, which are responsible for the astringency in the wine (McRae \& Kennedy, 2011). The alternative wines (w3 to w5) were found to contain much higher concentrations of flavan-3-ols and procyanidins than the traditional wines (w1 and w2). The latter only contained (+)-catechin and (-)-epicatechin monomers, procyanidins $\mathrm{B} 2$ and $\mathrm{B} 4$, and $\mathrm{B} 2-3-\mathrm{O}$-gallate dimer. Both monomers were present at very low concentrations (approximately $1.6 \mathrm{mg} / \mathrm{L}$ for $(+)$-catechin and $4.6 \mathrm{mg} / \mathrm{L}$ for (-)-epicatechin) relative to the alternative wines, where they exceeded $25 \mathrm{mg} / \mathrm{L}$ and reached levels of up to $44 \mathrm{mg} / \mathrm{L}$ for (-)-epicatechin and $93 \mathrm{mg} / \mathrm{L}$ for (+)-catechin. Similarly, the combined concentration of the two procyanidins (B2 + B4) in the traditional wines was below $4 \mathrm{mg} / \mathrm{L}$ and much lower than in the alternative wines (up to $17.6 \mathrm{mg} / \mathrm{L}$ ). The alternative wines also contained procyanidins B1 and B3, which were absent from the traditional wines. As a result of the low concentrations of the abovementioned flavanols in the traditional wines, B2-3-O-gallate dimer was the major component, with concentrations at the same level as those in the alternative wines. However, the latter also contained B2-3-O'-gallate and B1-3-O-gallate dimers. The fact that the flavan-3-ol fraction in the alternative wines contained many more compounds and at higher concentrations than those in the traditional wines suggests that these compounds become important substrates of polymerisation and/or degradation reactions during the sun drying of grapes or during their subsequent vinification. The traditional wines, with a lower concentration of these compounds, therefore exhibited a much higher browning index than the alternative wines.

In relation to flavonols, the traditional wines only contained the aglycones myricetin, quercetin, laricitrin, kaempferol, isorhamnetin and syringetin, all at very low concentrations. As can be seen in Table 3, quercetin was the major component in this fraction, with concentrations from 0.694 to $1.97 \mathrm{mg} / \mathrm{L}$. The alternative wines contained the aglycones myricetin, quercetin, kaempferol and isorhamnetin, as well as flavonol glycosides at much higher concentrations. The absence of flavonol glycosides and the presence of aglycones in wine are usually indicators of ageing, because under wine acid conditions, the glycosides are gradually hydrolysed to free aglycones (Jeffery et al., 2008). If flavonol glycosides, naturally present in the grapes, had been hydrolysed, the aglycone concentration should have been higher than it was in the commercial wines. Therefore, the degradation of this phenolic fraction must be considered as a potential cause of their substantially decreased concentrations. Also, the concentration of aglycones may have been further reduced by their low solubility in ethanol solutions such as wine, causing the formation of colourless crystals or yellow precipitates, detracting from the wine stability (Jeffery et al., 2008).

The analytical properties of the two types of wine were compared via principal component analysis (PCA), using the colour parameters and the concentrations of the different phenolic fractions. Fig. 4 shows the plane defined by the first two principal components (PCs), which jointly explained $93.1 \%$ of the overall variance in the process. The plane contains the scores or eigenvalues of the samples and the eigenvectors of the variables considered in the multivariate analysis. PC1, which accounted for $82.3 \%$ of the variance, clearly discriminated between wines obtained by different grape-drying methods. Thus, the wines from sun-dried grapes exhibited negative scores for this component, and those from chamber-dried grapes exhibited positive scores. The former exhibited higher hue values, higher contents of polymeric pigments, increased contributions of yellow (\%yellow) and the presence of hydroxycinnamic acids. By contrast, the alternative wines exhibited greater contributions of \%red and \%blue, and increased concentrations of monomeric anthocyanins, flavan-3-ols and flavonols.

In conclusion, the alternative wines are therefore analytically closer to typical red wines by their lower concentration of browning compounds, a high contribution of red and blue, and hence a more suitable colour than the commercial traditional wines. Also, the alternative wines were richer in phenolic compounds, particularly in monomeric anthocyanins, which give these wines their colour and are responsible for their antioxidant properties (Sun et al., 2011). In addition, the sensory properties of the alternative wines are likely to be highly acceptable for consumers. Based on previous works (Serratosa et al., 2008a), chamber drying has some other advantages over sun drying. Thus, controlled drying allows the use of grapes of variable ripening status in order to shorten the drying time needed to obtain raisins of similar sweetness and to increase must yield. A preliminary study confirmed that the alternative drying system was economically viable from a commercial point of view. Also, the controlled conditions of the chamber-drying process exclude the development of toxin-producing fungi, which is highly likely to occur during sun-drying of grapes (Serratosa 


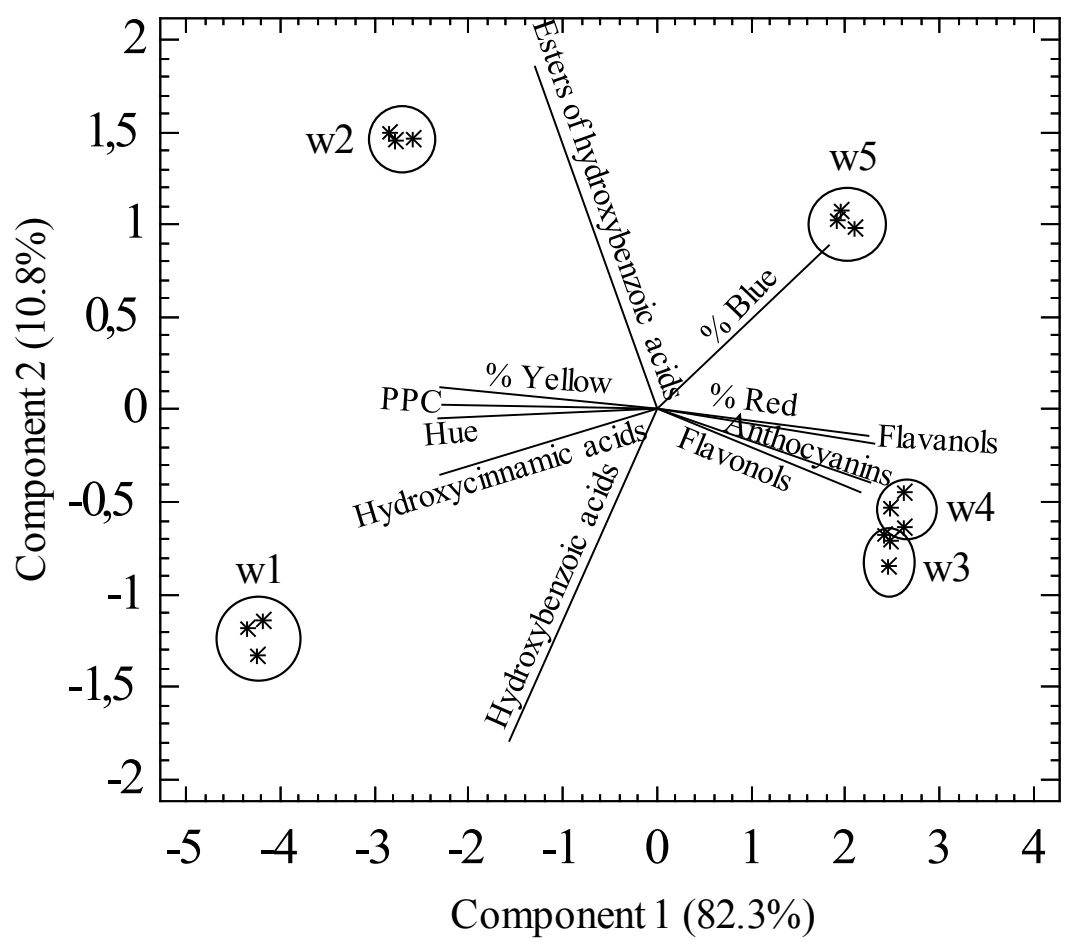

FIGURE 4

Principal component analysis of commercial (w1 and w2) and alternative sweet red wines (w3 to w5). Biplot representation of eigenvalues and eigenvectors.

et al., 2010). On the whole, the alternative winemaking process based on controlled chamber drying improved the quality of sweet red wines relative to commercial wines obtained by the traditional method.

\section{LITERATURE CITED}

Alen-Ruiz, F., Garcia-Falcon, M.S., Perez-Lamela, M.C., MartinezCarballo, E. \& Simal-Gandara, J., 2009. Influence of major polyphenols on antioxidant activity in Mencía and Brancellao red wines. Food Chem. 113, 53-60.

Bakker, J., Picinelli, A. \& Bridle, P., 1993. Model wine solutions: Colour and composition changes during ageing. Vitis 32, 111-118.

Bellicontro, A., De Santis, D., Botondi, R., Villa, I. \& Mencarelli, F., 2004. Different postharvest dehydration rates affect quality characteristics and volatile compounds of Malvasia, Trebbiano and Sangiovese grapes for wine production. J. Sci. Food Agric. 84, 1791-1800.

Chira, K., Jourdes, M. \& Teissedre, P.L., 2012. Cabernet Sauvignon red wine astringency quality control by tannin characterization and polymerization during storage. Eur. Food Res. Technol. 234, 253-261.

Downey, M.O., Dokoozlian, N.K. \& Krstic, M.P., 2006. Cultural practice and environmental impacts on the flavonoid composition of grapes and wine: A review of recent research. Am. J. Enol. Vitic. 57, 257-268.

Escribano-Bailon, T., Alvarez-Garcia, M., Rivas-Gonzalo, J.C., Heredia, F.J. \& Santos-Buelga, C., 2001. Color and stability of pigments derived from the acetaldehyde-mediated condensation between malvidin-3-Oglucoside and (+)-catechin. J. Agric. Food Chem. 49, 1213-1217.

Fischer, U. \& Noble, A.C., 1994. The effect of ethanol, catechin concentration, and $\mathrm{pH}$, on sourness and bitterness of wine. Am. J. Enol. Vitic. 45, 6-10.

Fulcrand, H., Benabdeljalil, C., Rigaud, J., Cheynier, V. \& Moutounet, M., 1998. A new class of wine pigments generated by reaction between pyruvic acid and grape anthocyanins. Phytochem. 47, 1401-1407.
Garcia-Puente Rivas, E., Alcalde-Eon, C., Santos-Buelga, C., RivasGonzalo, J.C. \& Escribano-Bailón, M.T., 2006. Behaviour and characterisation of the colour during red wine making and maturation. Anal. Chim. Acta 563, 215-222.

Genovese, A., Gambuti, A., Piombino, P. \& Moio, L., 2007. Sensory properties and aroma compounds of sweet Fiano wine. Food Chem. 103, 1228-1236.

Glories, Y., 1984. La couleur des vins rouges II. Mesure, origine et interprétation. Connaissances de la Vigne et du Vin 18, 253-271.

He, J., Carvalho, A., Mateus, N. \& Freitas, V., 2010. Spectral features and stability of oligomeric pyranoanthocyanins-flavanol pigments isolated from red wines. J. Agric. Food Chem. 58, 9249-9258.

Jackson, D.I. \& Lombard, P.B., 1993. Environmental and management practices affecting grape composition and wine quality - A review. Am. J. Enol. Vitic. 44, 409-430.

Jeffery, D.W., Parker, M. \& Smith, P.A., 2008. Flavonol composition of Australian red and white wines determined by high-performance liquid chromatography. Aust. J. Grape Wine Res. 14, 153-161.

Li, H., Guo, A. \& Wang, H., 2008. Mechanisms of oxidative browning of wine. Food Chem. 108, 1-13.

Lopez, P.A., Moreno, J. \& Medina, M., 1988. Caracterización del envero y madurez en la uva de Vitis vinifera Pedro Ximenez cultivada en la zona Montilla-Moriles. Revista de Agroquimica y Tecnologia de Alimentos 28, 274-284.

Marquez, A., Dueñas, M., Serratosa, M.P. \& Merida, J., 2012a. Formation of vitisins and anthocyanin-flavanol adducts during red grape drying. J. Agric. Food Chem. 60, 6866-6874.

Marquez, A., Serratosa, M.P., Lopez-Toledano, A. \& Merida, J., 2012 b. Colour and phenolic compounds in sweet red wines from Merlot and Tempranillo grapes chamber-dried under controlled conditions. Food Chem. 130, 111-120. 
McRae, J.M. \& Kennedy, J.A., 2011. Wine and grape tannin interactions with salivary proteins and their impact on astringency: A review of current research. Molecules 16, 2348-2364.

Monagas, M., Martin-Alvarez, P.J., Bartolome, B. \& Gomez-Cordoves, C., 2006. Statistical interpretation of the colour parameters of red wines in function of their phenolic composition during aging in bottle. Eur. Food Res. Technol. 222, 702-709.

Perez-Magariño, S., Ortega-Heras, M., Cano-Mozo, E. \& Gonzalez-Sanjose, M.L., 2009. The influence of oak wood chips, micro-oxygenation treatment, and grape variety on colour, and anthocyanin and phenolic composition of red wines. J. Food Comp. Anal. 22, 204-211.

Petropoulos, S., Kallithraka, S. \& Paraskevopoulos, I., 2011. Influence of some viticultural practices on the polyphenolic content of wines produced from cv. Agiorgitiko (Vitis vinifera L.). J. Int. des Sciences de la Vigne et du Vin, 45, 235-243.

Prado, R.A., Yuste-Rojas, M., Sort, X., Andrea-Lacueva, C., Torres, M. \& Lamuela- Raventos, R.M., 2007. Effect of soil type on wines produced from Vitis vinifera L. cv. Grenache in commercial vineyards. J. Agric. Food Chem. 55, 779-786.

Rivero-Perez, M.D., Perez-Magariño, S. \& Gonzalez-San Jose, M.L., 2002. Role of melanoidins in sweet wines. Anal. Chim. Acta 458, 169-175.

Rolle, L., Giordano, M., Giacosa, S., Vincenzi, S., Rio Segade, S., Torchio, F., Perrone, B. \& Gerbi, V., 2012. CIEL*a*b* parameters of white dehydrated grapes as quality markers according to chemical composition, volatile profile and mechanical properties. Anal. Chim. Acta 732, 105-113.

Ruiz, M.J., Zea, L., Moyano, L. \& Medina, M., 2010. Aroma active compounds during the drying of grapes cv. Pedro Ximenez destined to the production of sweet Sherry wine. Eur. Food Res. Technol. 230, 429-435.
Serratosa, M.P., Lopez-Toledano, A., Medina, M. \& Merida, J., 2008a. Drying of Pedro Ximenez grapes in chamber at controlled temperature and with dipping pretreatments. Changes in the color fraction. J. Agric. Food Chem. 56, 10739-10746.

Serratosa, M.P., López-Toledano, A., Mérida, J. \& Medina, M., 2008b. Changes in color and phenolic compounds during the raisining of grape cv. Pedro Ximenez. J. Agric. Food Chem. 56, 2810-2816.

Serratosa, M.P., Lopez-Toledano, A., Millan, C., Medina, M. \& Merida, J., 2010. Changes of ochratoxin A in grapes inoculated with Aspergilus carbonarius and subjected to chamber-drying under controlled conditions. J. Agric. Food Chem. 58, 11907-11912.

Serratosa, M.P., Marquez, A., Lopez-Toledano, A. \& Merida, J., 2012. Sensory analysis of sweet musts in Pedro Ximenez cv. grapes dried using different methods. S. Afr. J. Enol. Vitic. 33, 14-20.

Soto-Vazquez, E., Rio-Segade, S. \& Orriols-Fernandez, I., 2010. Effect of the winemaking technique on phenolic composition and chromatic characteristic in young red wines. Eur. Food Res. Technol. 231, 789-802.

Sun, B., Neves A.C., Fernandes T.A., Fernandes A.L., Mateus N., De Freitas V., Leandro C. \& Spranger, M.I., 2011. Evolution of phenolic composition of red wine during vinification and storage and its contribution to wine sensory properties and antioxidant activity. J. Agric. Food Chem. 59, 65506557.

Vidavalur, R., Otani, H., Singal, P.K. \& Maulik, N., 2006. Significance of wine and resveratrol in cardiovascular disease: French paradox revisited. Exp. Clin. Cardiol. 11, 217-225. 